\title{
Astrophysical Constraints on Modifying Gravity at Large Distances
}

\author{
A. Aguirre ${ }^{a}{ }^{\text {C.P. Burgess }},{ }^{a, b}$ A. Friedland ${ }^{a}$ and D. Nolte ${ }^{a}$ \\ ${ }^{a}$ School of Natural Sciences, Institute for Advanced Study, Princeton, New Jersey 08540, USA \\ ${ }^{b}$ Physics Department, McGill University, 3600 University St., Montréal, Québec, Canada, H3A $2 T 8$.
}

\begin{abstract}
Recently, several interesting proposals were made modifying the law of gravity on large scales, within a sensible relativistic formulation. This allows a precise formulation of the idea that such a modification might account for galaxy rotation curves, instead of the usual interpretation of these curves as evidence for dark matter. We here summarize several observational constraints which any such modification must satisfy, and which we believe make more challenging any interpretation of galaxy rotation curves in terms of new gravitational physics.
\end{abstract}

PACS numbers:

\section{INTRODUCTION}

The observed flatness of galaxy rotation curves are used as important evidence for the existence of dark matter on galactic scales. These observations thus form a keystone of the structure of inferences that produce the current cosmological paradigm, which requires the vast majority of the matter in the universe to consist of unknown, dark forms. Although this paradigm has received considerable support from recent observations, this support has been at the expense of simplicity, since the evidence now requires two forms of dark matter: both the conventional sort plus the newly-discovered 'dark energy' [1.2.

The validity of the Newton-Einstein laws of gravity on galactic scales is an important component of the standard reasoning, and one for which the direct observational support is comparatively weak. Although much less work has been done to explore possible modifications of gravity on large scales than has been invested in dark matter models, over the years a literature on the subject has emerged [3]- [10]. Among the variants is a reasonably simple modification of Newtonian dynamics which describes galaxy rotation curves quite well, without requiring the existence of dark matter 11, 14].

Much of this work has remained outside of the mainstream for various reasons. Some proposals invent ad-hoc nonrelativistic potentials without addressing how these potentials might arise within the context of relativistic field theory. This is not a small omission, because it is precisely the issue of relativistic consistency which makes modifying physics at large distances difficult. Although it has long been known that the laws of gravity are likely to receive modifications on short distance scales, general constraints like unitarity and lorentz invariance in weak fields limit what can be done on very large scales. These arguments seem to restrict any such modification to take the form of supplementing the graviton with fields describing various low-spin degrees of freedom 15, leading

\section{McGill-01/07}

to scalar-tensor-Maxwell kinds of theories at long distances. . While some of the proposed modifications to gravity fall into this category it has proven difficult to reproduce the galactic rotation curves with these theories without also running afoul of other constraints [16].

The situation may now be changing, with several recent claims that self-consistent particle-physics models permit the law of gravity to be modified on large scales. Several recent proposals do so using constructions 17] within the braneworld picture [18, 19], although none of these has yet tried to achieve acceptable phenomenology at large distances. Ref. 20] aims to describe spiral galaxy rotation curves assuming the existence of extremely light four-dimensional fields trapped onto galactic-sized defects. These new developments motivate re-examining the astrophysical constraints on modifications to gravity.

It is not our purpose in this note to present another modification of gravity at large distance scales. Rather, we put aside general issues of principle, and indicate what targets proposed models must hit in order to agree with the extensive astrophysical data on galaxy dynamics. We shall argue here that the data pose a significant challenge which must be met by any hypothetical modifications to gravity, and we believe none of the extant proposals completely meet this challenge.

The constraints come in several types. First, the systematics of galaxy rotation curves are observationally found to be described by a universal shape which depends only on the galaxy luminosity and visible size. In particular, the huge range in sizes of observed galaxies precludes theories which modify gravity at a fixed distance for all galaxies. Second, the galactic gravitational potential for spiral galaxies like the Milky Way is mea-

\footnotetext{
${ }^{*}$ These do not include higher-derivative theories, which generically have runaway modes which violate the unitarity and stability constraints.
} 
sured in 3 dimensions, not just in the plane of the galactic disk. Finally, additional constraints come from the TullyFisher relation and the x-ray observations of gas in galaxy clusters. Some of these constraints have been raised elsewhere as objections to various specific proposed modifications [21,22.

We emphasize that our purpose in making this argument is not to argue that phenomenologically-successful modifications to gravity cannot be made (although this may in the end prove to be true). Rather, we intend to provide a convenient summary of the astrophysical constraints which are relatively unfamiliar to the particle physics and quantum gravity communities.

\section{CONSTRAINTS}

We now summarize the most serious astrophysical constraints, whose successful description we claim to be a necessary (though not sufficient) condition for the success of any putative realistic modification to gravity at large distances. Since most of the proposed modifications are motivated to describe galaxy rotation curves, we describe these first before turning to the other constraints.

1. Rotation Curve Systematics: Observations on many galaxies indicate rotation velocities, $v_{\text {rot }}$, which for sufficiently large distance, $r$, from the galactic center, become independent of $r$. This is to be contrasted with the Keplerian form, $v_{\text {rot }}^{2}=G M / r$, which would be expected for circular orbits well outside of a concentrated mass distribution, and which Newton's Law would also approximately predict for galaxies sufficiently far outside the visible material (in the absence of dark matter).

A common way to account for this amongst proposed modifications of gravity is to have the gravitational potential be modified from the Newtonian form $-G M / r$ to a new form: $-G M / r+U(r)$. (Proposals for $U(r)$ include linear [9,6], logarithmic [4,20] or Yukawa-type [7] functions of $r$, or have it interpolate between different asymptotic Newtonian regimes with different values for $G$ [8.5.) The successful comparison of General Relativity with solar-system and terrestrial measurements 22] is then accounted for by ensuring $U(r)$ becomes sufficiently small when $r<r_{0}$, where $r_{0}$ is a new fundamental characteristic distance scale.

For the vast majority of models, the modified force is itself proportional to $M-$ i.e. $U(r)=M u(r)$ with $u(r)$ an $M$-independent function - because it is obtained by the superposition of forces exerted by the individual particles of which the galaxy is made. It follows that any such model crosses over from Newtonian to exotic force laws for a radius, $r_{0}$, which is independent of the properties of the galaxy involved. If

Any model for which this crossover radius is universal - regardless of the details of the force law responsible does not provide a good description of the systematics of galaxy rotation curves. It does not because it predicts the scale, $r_{0}$, to be independent of the luminosity of the galaxy. This conflicts with observations, for which the transition radius (at which the rotation curves deviate from the predictions of Newtonian gravity applied to the visible mass) occurs at radii which are correlated with the galaxy's optical size, f $R_{\text {opt }}$. Since 'non-Newtonian' rotation curves have been seen in galaxies that range over several orders of magnitude in overall luminosity, the observed transition radius (and optical size) varies over an order of magnitude, from a few to a few tens of kiloparsecs.

The data indicate that when a galaxy's rotation speed is plotted against $r / R_{\text {opt }}$, the resulting curve is a universal function which depends only on the total luminosity of the galaxy (see Eq. (14) and Fig. 10 in 23]). In galaxies with low luminosities, the contribution of dark matter becomes important well inside the optical radius, while in highest luminosity (very large) galaxies luminous matter adequately describes the rotation curves to at least $r / R_{\text {opt }} \sim 1$. This behavior is incompatible with modifications of the gravitational force law at a fixed distance from the source.

To be completely concrete, consider the case of two galaxies, N598 and N801 (with data taken from [23]). The data show that the rotation curve of the lowluminosity galaxy, N598, would demand the modification of gravity at distances less than its optical radius, which is $3.9 \mathrm{kpc}$. This would cause a dramatic effect on the rotation curve of the high-luminosity N801, well inside of its optical radius of $38.4 \mathrm{kpc}$. No such distortion is observed. We see that the larger galaxies would exhibit more - not less - dark matter than the small ones at a given fraction of their optical radius, were the law of gravity modified at a fixed distance.

The existence of modifications to gravity which can successfully ensure that the modifications become important at distances which depend on the galaxy involved is demonstrated by the proposal called MOND [1] 13. MOND's success in describing galaxy rotation curves relies on its modifying Newtonian dynamics at a minimum acceleration, rather than modifying Newton's Force Law above a maximum distance. That is, the modification of rotation curves from the Newtonian prediction does

\footnotetext{
${ }^{\dagger}$ Ref. 10 avoids this conclusion by adding two additional force terms, one depending on $M$ and one depending solely on the distance from the galaxy center.

${ }^{\ddagger}$ We use $R_{\text {opt }}=3.2 R_{D}$, where $R_{D}$ is the exponential disk scale length, in accordance with the definition of Ref. 23.
} 
not happen at the same distance from the center of all galaxies, but occurs at a radius for which the Keplerian acceleration is of order $a_{0} \sim 10^{-10} \mathrm{~m} / \mathrm{s}^{2}$ [14.24. Indeed, part of what is remarkable about the observational success that MOND has in describing observations is the successful fitting of the rotation curves of many different kinds of galaxies in terms of a single function describing the crossover from Newton's law at high acceleration $a$ to its modified version at small acceleration around $a_{0}$. Best fits also give very reasonable values for the mass-toluminosity ratio, $\Upsilon=M / L$ (and so does not introduce a new reason to require a new kind of dark matter).

2. The Tully-Fisher Relation and $M / L$ : Besides predicting flat rotation curves, any modification of gravity should also account for the dependence of the rotation curves on galaxy luminosity. Part of this dependence is summarized by the Tully-Fisher relation [25], which expresses an observational fact: the luminosity, $L$, of a spiral galaxy is tightly correlated with the asymptotic limit of its rotation velocity, $v_{\infty}=\lim _{r \rightarrow \infty} v_{\text {rot }}(r)$, according to

$$
L \propto v_{\infty}^{\alpha}
$$

with $\alpha \approx 4$ 26].

While the Tully-Fisher relation (and particularly its small scatter) is not completely understood within the dark-matter picture, it can be roughly derived in simple models where galactic disks collapse with conserved angular momentum within dark matter halos of a form predicted by numerical simulations [27]. But any proposal to replace dark matter with a modification of gravity should be able to do better, by directly yielding the T-F relation once the mass-to-light ratio $\Upsilon$ of the matter constituting the galaxies is specified. Conversely, to satisfy the Tully-Fisher relation any modified gravity theory will predict a specific form for $\Upsilon(L)$, and this prediction can be tested.

For example, all models which modify the positiondependence of the gravitational potential at large $r$ by supplementing the Newtonian term with $M u(r)$ predict $v_{\infty}^{2} / M$ to be purely a function of $r$ for circular orbits. If $u(r)$ for a given model is designed to approach a constant for large $r$ (to describe the observed flat rotation curves), then that model predicts $M \propto v_{\infty}^{2}$ [12], which is consistent with the Tully-Fisher relation only if $\Upsilon=M / L \propto L^{-1 / 2}$. This relation is, however, strongly constrained because $\Upsilon$ can be estimated both theoretically and observationally for baryonic matter in galaxies over a wide range in luminosity.

Theoretically, $\Upsilon \approx(0.5-4) M_{\odot} / L_{\odot}$ in stellar material is predicted by stellar evolution models that can reasonably reproduce the colors and spectra of observed spiral galaxies [28]. Similar values can also be directly measured in the centers of both high- and moderate- luminosity galaxies, where stars are expected to dominate the mass (and where Newtonian gravity would presumably hold) [29]. More generally, stars are observed to dominate the mass of known baryonic matter in galaxies - only in the smallest dwarf galaxies does the mass in gas approach or (marginally) exceed that in stars [30].

Thus $\Upsilon \sim(0.5-4) M_{\odot} / L_{\odot}$ is expected to hold for spiral disks of all luminosities. This does not pose a problem for MOND (for example), which predicts $L \propto v_{\infty}^{4}$ and therefore generally predicts $\Upsilon$ to be independent of $L$ and to lie within this range 31]. It is problematic for any theory predicting a varying $\Upsilon(L)$, since the T-F relation is observed to hold over four decades in $L$ [30]. For example, the prediction $\Upsilon \propto L^{-1 / 2}$ generic to many models implies that $\Upsilon$ must be 100 times larger in the least luminous observed galaxies than in the most luminous ones. Since the stellar populations of dwarf and giant galaxies probably have similar $\Upsilon$, the T-F relation can be fit only by assuming that $\sim 99 \%$ of the mass of the least luminous galaxies is in an unknown form (i.e. neither observed stars or atomic or molecular gas). Although it may be possible to invent forms of baryonic matter in which such large amounts of mass might be hidden, this would be rather unsatisfying in a model constructed to remove the need for dark matter.

3. Galaxy Potentials in Three Dimensions: Since Newton's law in $d$ space dimensions implies a gravitational potential which varies as $(1 / r)^{d-2}$, it is tempting to try to obtain logarithmic potentials - and so constant Keplerian velocities - by trapping the field which mediates this force in some way to two space dimensions (for which Newton predicts a logarithmic dependence). At first sight this is a very attractive possibility, since if a field contributing to long-distance interactions is trapped on a surface of thickness $\ell \sim$ several kiloparsecs, then interparticle potentials vary like $1 / r$ when $r \ll \ell$, but cross over (within the plane) to $\log r$ dependence for $r \gg \ell$. (See ref. [20] for a recent attempt to construct such a mechanism.)

A fundamental prediction of any such mechanism is that galactic rotation curves should look flat in the directions along the trapping surface, but the galactic gravitational well should be Newtonian when examined in directions perpendicular to this surface. Putting aside questions of how elliptical galaxies (which are not flattened and yet also appear to have dark matter [32]) might fit into such a scenario, there are several pieces of evidence concerning our own galaxy which prospective models must confront.

- Globular Clusters: Globular clusters are well-defined clusters of stars which are distributed in an approximately spherically-symmetric way about the galactic center. They provide an important constraint on models of the galaxy because: $(i)$ they are not confined to the plane of the spiral arms; (ii) they are distributed out to 
$40 \mathrm{kpc}$ from the galactic center; and (iii) at least 26 of the roughly 150 known globular clusters have measurable proper motions relative to extragalactic background objects [33, 34], and so have known velocities and positions.

The constraint on galaxy models arises as follows. Given their known positions and velocities, it is possible to reconstruct the galactic orbits of many globular clusters given a model of the galactic gravitational potential. In particular, for many models - including those for which the galactic mass does not extend out to 40 $\mathrm{kpc}$ or so - the orbital apocenters (i.e. those points most distant from the galactic center) lie 50 to $80 \mathrm{kpc}$ from the galactic centers. Since most of the time in an orbit is spent near the orbital apocenter, it is statistically very unlikely to find less than a handful of clusters further than $40 \mathrm{kpc}$ from the galactic center, as is observed [33].

- The Magellanic Stream: The Magellanic Stream is a trail of neutral hydrogen which extends in a great circle more than $100^{\circ}$ across the sky, starting at the Magellanic Clouds. This hydrogen is most likely gas which has been stripped from the Clouds due to tidal forces, and because the Milky Way's gravitational potential is much deeper than that of the Clouds, the Stream is distributed along the orbit of the Magellanic Clouds themselves. This picture is consistent with the measured infall velocity of the Stream, which varies nearly linearly as a function of distance from the Clouds, reaching $-200 \mathrm{~km} / \mathrm{s}$ at the end furthest from the Clouds. The line-of-sight velocity of the center of mass of the Magellanic Clouds themselves is $61 \mathrm{~km} / \mathrm{s}$, indicating that the Clouds are probably near the pericenter - closest approach - of their orbit, which must be quite eccentric.

The constraint on the Galactic potential is obtained by requiring the existence of an orbit with a pericenter of roughly $q=50 \mathrm{kpc}$ and on which radial velocities as large as $200 \mathrm{~km} / \mathrm{s}$ are obtained 35. This puts strong constraints on models with all of the Milky Way's mass localised where the luminous matter is, and in which gravity is Newtonian out of the galactic plane, since for these the Stream motion should be approximately Keplerian. Since the maximum radial velocity of a bound Keplerian orbit with pericenter $q$ is $v_{\max }=(G M / 2 q)^{\frac{1}{2}}$, for $q=50$ $\mathrm{kpc}$, the observed $200 \mathrm{~km} / \mathrm{s}$ infall of Stream material implies a galactic mass $M>9 \times 10^{11}$ solar masses. This is an order of magnitude larger than the mass obtained by counting the luminous matter in the disk, but is consistent with the amount of dark matter required to account for rotation curves.

- Local Group Galaxies: Constraints similar in spirit to, but weaker than, the one just described can be obtained from the dwarf galaxies which make up much of our local galactic neighborhood. A number of arguments indicate that these are bound to our galaxy [35] and their observed distances and radial velocities constrain the shape and depth of the galaxy's gravitational well. In particular, the large speed of some of these dwarfs (such as Leo I, Pal 14 and Eridanus) can only be bound by the presence of much more mass than is visible if the out-of-plane gravitational field is Newtonian.

4. The Dynamics and Structure of Clusters of Galaxies: As first notice by Zwicky [36], the visible mass in clusters of galaxies is entirely too small to bind them, given the observed velocity dispersion (several hundred $\mathrm{km} / \mathrm{s}$ ) in their component galaxies; this was the first strong evidence for dark matter (or modified gravity) in cosmology, and is yet stronger today. X-ray measurements of clusters have revealed that their observable baryonic masses are dominated by hot gas of temperature $\sim 1-10 \mathrm{keV}$, which also implies (using standard gravity) a binding mass well above that observed in galaxies and the hot gas itself.

It is instructive to derive this inconsistency explicitly, in order to see the logic of the constraints posed on dark matter and on modifications of gravity. Consider, then, the equation of hydrostatic equilibrium in a spherical system (which describes intracluster gas well):

$$
\frac{1}{\rho} \frac{d P}{d r}=-a(r)
$$

where $\rho$ and $P$ are the density and pressure of the gas, and $a$ is the inward gravitational acceleration at radius $r$. Using the ideal gas equation of state, this can be re-written in terms of the gas temperature $T$ and mean molecular weight, $\mu \sim 0.6$ (in units of the proton mass, $\left.m_{p}\right)$ :

$$
\frac{d \log \rho}{d \log r}+\frac{d \log T}{d \log r}=-\frac{r}{T}\left(\frac{\mu m_{p}}{k}\right) a(r),
$$

where $k$ is Boltzmann's constant. Clusters are observed to have temperature profiles, $T(r)$, which are roughly constant outside of their cores. The density profile of the observed gas at large radii roughly follows a power law, $\rho \propto r^{\alpha}$, with index $-2 \lesssim \alpha \lesssim-1.5$ [37]. Using these, the fact that the gas dominates the cluster's baryonic mass, and Newton's expression for $a(r)$ in the absence of dark matter gives

$$
k T \approx(1.3-1.8) \mathrm{keV}\left(\frac{M_{r}}{10^{14} M_{\odot}}\right)\left(\frac{1 \mathrm{Mpc}}{r}\right)
$$

for the baryonic mass within the central $1 \mathrm{Mpc}$ of a typical rich cluster, where $M_{r}$ is the mass enclosed within $r$. The disparity between this estimate and the corresponding observed temperature $\approx 10 \mathrm{keV}[38$ ] indicates the need for dark matter or modified gravity.

Similar reasoning provides a strong constraint on any modifications of gravity, for which the only matter present is visible and $a(r)$ is calculable. Most generally, Eq. (3) implies an approximate relation between the total mass, outer radius, and average temperature of the cluster. For a given gravity theory, this relation can be 
checked by comparing to observed masses, temperatures, and sizes of observed clusters, as we have done for Newtonian gravity above. A useful compilation of applicable cluster data is given in [38]. MOND can apparently survive this comparison [39], as can the standard CDM cosmology with $\sim 5-10$ times as much dark matter as baryonic matter, whereas (for example), the construction of 20] would not account for the high cluster temperatures (and hence would still require the standard amount of dark matter) because most cluster gas would not lie within the domain walls and would hence feel only the usual Newtonian gravitation of the visible matter.

A stronger constraint can be deduced using observed density, mass and temperature profiles of individual clusters. If gravity depends only on the visible mass, the observed density profile $\rho(r)$ and enclosed mass profile $M_{r}$ of a given cluster can be used to directly predict its temperature profile $T(r)$ in a given gravity theory using Eq. (3), and the profile compared to the measured one. Roughly speaking, the combination $r a(r)$, which is proportional to $T(r)$ (since $d \ln \rho / d \ln r$ and $T$ are observed to be approximately constant) should be nearly independent of $r$, as $T(r)$ is observed to be. This technique is illustrated in detail in [39], where it is shown that MOND predicts rising radial temperature profiles that disagree badly with the observations (which show that clusters are roughly isothermal).

Other modifications of gravity may encounter similar difficulties. Consider, for example, the hydrostatic equilibrium of cluster gas when large- $r$ gravity is dominated by a linear potential [9,10]. Since linear potentials are sensitive to the mass distribution at large radius, we will assume the cluster gas to not extend beyond an outer radius, $R$. For simplicity of analysis we bracket the real mass density for $r<R$ between two extremes: it is taken to be concentrated at radius $r=0$, or to be uniform within $R$. Under these assumptions we find that for $r \ll R, r a(r)$ respectively grows either linearly or quadratically with $r$. Provided only that a more realistic treatment lies between these two extremes, we see that $T(r)$ is predicted to increase linearly to quadratically with $r$. Such a prediction would be ruled out by the observations, although a more detailed study would be required to make this conclusion fully quantitative.

Finally, we note that cluster mass estimates using galactic dynamics or the X-ray gas are consistent (to within a factor of two) with masses determined by weak gravitational lensing of background galaxies around the clusters 40]. Thus any alternative gravity theory must

\footnotetext{
${ }^{\S}$ Given the extreme sensitivity of the interaction energy to matter at arbitrarily large radii, it is unclear how to compute the characteristics of a given physical system with a linear potential without neglecting this contribution.
}

also predict gravitational lensing in a way that allows this. This constraint was used, for example, by [4] to rule out their model, and provides difficulties for MOND in cluster cores [14].

It would be wonderful to discover new gravitational physics from astrophysical observations, and it is intriguing that current braneworld models seem to imply that long-distance gravitational physics can be much richer than had been previously thought. In order for any such physics to provide a viable alternative to dark matter it must satisfy the above constraints, and a theory that successfully does so would be of sufficient interest to merit a more detailed investigation (including detailed rotationcurve fitting, lensing calculations, predictions concerning the formation of structure, cosmological predictions, etc.). We hope our summary of these constraints will further stimulate thinking about the relation between astrophysical puzzles and current ideas in high-energy physics.

We are grateful to Gregory Gabadadze for his valuable comments. This research was partially funded by grants from F.C.A.R (Québec), N.S.E.R.C. (Canada), as well as the Ambrose Monnell and the W.M. Keck Foundations.

[1] P. J. E. Peebles, P.A.S.P 111, 274 (1999); M. S. Turner, P.A.S.P 111, 264 (1999); A. Dekel, D. Burstein, and S. D. M. White, 1997, Critical Dialogues in Cosmology, p. 175 .

[2] S. Perlmutter et al, Nature 391, 51 (1998), [astro-ph/9712212]; A. G. Riess et al, Astron. J. 116, 1009 (1998), [astro-ph/9805201].

[3] A. Finzi, Mon. Not. R. Astron. Soc. 127, 21 (1963); J. E. Tohline, Internal kinematics and dynamics of galaxies, Dordrecht, Reidel (1983), p. 205; J. Bekenstein, Phys. Lett. B. 202, 497 (1988); R. H. Sanders, Mon. Not. R. Astron. Soc. 241, 135 (1989); H. J. Fahr, Astron. Astrophys. 236, 86 (1990); E. Battaner, J. L. Garrido, M. Membrado, and E. Florido, Nature 360, 652 (1992); Gessner E., Astrophys. \& Space Sci. 194, 197 (1992).

[4] W. H. Kinney and M. Brisudova, astro-ph/0006453, to appear in Proc. of the 15th Florida Workshop in Nonlinear Astronomy and Physics, 'The Onset of Nonlinearity'.

[5] I. T. Drummond, Phys. Rev. D 63, 043503 (2001), astro-ph/0008234.

[6] C. E. Carlson and E. J. Lowenstein, astro-ph/9602099.

[7] R. H. Sanders, Astron. Astrophys. 136, L21 (1984); D. H. Eckhardt, Phys. Rev. D 48, 3762 (1993).

[8] T. Goldman, J. Pérez-Mercader, F. Cooper, M. MartinNieto, Phys. Lett. B281, 219 (1992); O. Bertolami and J. Garcia-Bellido, Int. J. Mod. Phys. D 5, 363 (1996) astro-ph/9502010].

[9] P. D. Mannheim and D. Kazanas, Astrophys. J. 342, 635 (1989). 
[10] P. D. Mannheim and J. Kmetko, astro-ph/9602094; P. D. Mannheim, Astrophys. J. 479659 (1997).

[11] M. Milgrom, Astrophys. J. 270, 384 (1983).

[12] M. Milgrom, Astrophys. J. 270, 371 (1983).

[13] M. Milgrom, Astrophys. J. 270, 365 (1983).

[14] For a clear summary, with references, see M. Milgrom, astro-ph/9810302.

[15] S. Weinberg, The Quantum Theory of Fields, Vol. I, Cambridge University Press; S. Weinberg and E. Witten, Phys. Lett. B96 59 (1980).

[16] J.D. Beckenstein and M. Milgrom, Astrophys. J. 286, 7 (1984); J. D. Bekenstein and R. H. Sanders, Astrophys. J. 429, 480 (1994) astro-ph/9311062; R. H. Sanders Astrophys. J. 480, 492 (1997); V. V. Zhytnikov and J. M. Nester, Phys. Rev. Lett. 73, 2950 (1994).

[17] I.I. Kogan, S. Mouslopoulos, A. Papazoglou, G.G. Ross and J. Santiago, Nucl. Phys. B584, 313 (2000); R. Gregory, V. A. Rubakov and S. M. Sibiryakov Phys. Rev. Lett. 84, 5928 (2000) [hep-th/0002072]; C.Csáki, J. Ehrlich, T.J. Hollowood Phys. Rev. Lett. 84, 5932 (2000) hep-th/0002161, Phys. Lett. B481, 107 (2000) hep-th/0003020; G. Dvali, G. Gabadadze and M. Porrati, Phys. Lett. 484, 129 (2000) [hep-th/0003054]; JHEP 0007, 056 (2000); [hep-th/0004028]; Phys. Lett. B485, 208 (2000) hep-th/0005016]; C. Deffayet, Phys. Lett. B502, 199 (2001) [hep-th/0010186]; C. Csaki, J. Erlich, T.J. Hollowood and J. Terning Phys. Rev. 63, 065019 (2001) hep-th/0003076; G. Dvali and G. Gabadadze, Phys. Rev. D63, 065007 (2001) hep-th/0008054]; M. Carena, A. Delgado, J. Lykken, S. Pokorski, M. Quiros and C.E.M. Wagner, [hep-ph/0102172]; G. Dvali, G. Gabadadze, M. Kolanović and F. Nitti, hhep-ph/0102216.

[18] N. Arkani-Hamed, S. Dimopoulos and G. Dvali, Phys. Lett. B429, 263 (1998) [hep-ph/9803315, Phys. Rev. D59, 086004 (1999) [hep-ph/9807344] I. Antoniadis, N. Arkani-Hamed, S. Dimopoulos and G. Dvali, Phys. Lett. B436, 257 (1998) [hep-ph/9804398]; P. Hořava and E. Witten, Nucl. Phys. B475, 94 (1996) hep-th/9603142, Nucl. Phys. B460, 506 (1996) hep-th/9510209]; E. Witten, Nucl. Phys. B471, 135 (1996) [hep-th/9602070]; J. Lykken, Phys. Rev. D54, 3693 (1996) [hep-th/9603133; I. Antoniadis, Phys. Lett. B246, 377 (1990).

[19] L. Randall and R. Sundrum, Phys. Rev. Lett. 83, 3370 (1999) [hep-ph/9905221], Phys. Rev. Lett. 83, 4690 (1999) [hep-th/9906064].

[20] G. Dvali, G. Gabadadze and M. Shifman, astro-ph/0102422.

[21] R.H. Sanders, Mon. Not. R. Astr. Soc. 223, 539 (1986); C. Talmadge, J.-P. Berthias, R.W. Hellings, and E.M. Standish, Phys. Rev. Lett. 61, 1159 (1988); B. McFarland, Mon. Not. R. Astron. Soc. 242, 478 (1990); D. Scott, M. White, J.D. Cohn and E. Pierpaoli, astro-ph/0104435.

[22] C.M. Will, The Confrontation between General Relativity and Experiment, to appear in Living Reviews in Relativity,gr-qc/0103036; C.M. Will, Theory and Experiment in Gravitational Physics, 2nd Edition, Cambridge University Press, Cambridge (1993).

[23] M. Persic, P. Salucci and F. Stel, Mon. Not. R. Astron.
Soc. 281, 27 (1996); Erratum-ibid. 283, 1102 (1996).

[24] R.H. Sanders, Astrophys. J. 473, 4117 (1996).

[25] R.B. Tully and J.R. Fisher, Astron. Astrophys. 54, 661 (1977).

[26] While the slope $\alpha$ of the T-F relation varies from $\sim 2-4$ in different determinations, most of this variation results from the wavelength used; at shorter wavelengths where dust extinction and variations in mass-to-light ratio due to bright, young, hot stars are more important, $\alpha$ is smaller. T-F relations in infrared (e.g., I or K) bands are less subject to dust extinction and more accurately relate mass to rotation speed. Probably the two highest quality T-F relation determinations find that $\alpha=4.2$ in K-band and $\alpha=4.0$ in I-band; see, respectively, F. van den Bosch, Astrophys. J. 530, 177 (2000); S. Sakai et al, Astrophys. J. 529, 698 (2000).

[27] S. D. M. White, The Physical Origin of Galaxy Scaling Relation, in Galaxy Scaling Relations, ed. L.N. Da Costa, A. Renzini (Springer-Verlag), 1997.

[28] A. G. Bruzual and S. Charlot, Astrophys. J. 405, 538 (1993).

[29] C. Ratnam and P. Salucci, astro-ph/0008121.

[30] G. Gavazzi, D. Pierini, and A. Boselli, Astron. Astrophys 312, 397 (1996).

[31] W. J. G. de Blok and S. S. McGaugh, Astrophys. J. 508, 132 (1998).

[32] Elliptical galaxies lack the large quantities of cold gas used in spirals to observe rotation curves at large radii, so knowledge of elliptical galaxy kinematics at large radii is weaker. However, studies of globular clusters, gravitational lensing and X-ray emitting gas show in most cases that dark matter (or modified gravity) is required. For a good review of inferences of dark matter in elliptical galaxies, see M. Loewenstein and R. E. White, Astrophys. J. 518, 50 (1999).

[33] B. Dauphole and J. Colin, Astron. Astrophys. 300, 117 (1995).

[34] T. Bridges, in Galaxy Dynamics, ASP Conference Series vol. 182 (San Francisco: ASP), edited by David R. Merritt, Monica Valluri, and J. A. Sellwood, 1999, p. 415; T. J. Bridges et al, Mon. Not. R. Astron. Soc. 284, 376 (1997);

A. J. Romanowsky, Halo Dynamics of Elliptical Galaxies, in the proceedings of the American Astronomical Society Meeting 196, No. 16.01, May 2000;

A. J. Romanowsky and C. S. Kochanek, Halo Dynamics of $M 8^{7}$, in the proceedings of Dynamics of Galaxies: from the Early Universe to the Present, 15th IAP meeting, 1999, Eds.: Francoise Combes, Gary A. Mamon, and Vassilis Charmandaris ASP Conference Series, Vol. 197, ISBN: 1-58381-024-2, 2000, p. 401.

[35] M. Fich and S. Tremaine, The mass of the Galaxy, Annual review of astronomy and astrophysics Vol. 29, Palo Alto, CA, Annual Reviews, Inc., 1991, p. 409-445.

[36] F. Zwicky, Helvetic Physica Acta 6, 110 (1933).

[37] G. Cirimele, R. Nesci, and D. Trevese, Astrophys. J. 475, 11 (1997).

[38] J. Mohr, B. Mathiesen, and A.E. Evrard, Astrophys. J. 517, 627 (1999).

[39] A. Aguirre, J. Schaye, and E. Quataert, in preparation

[40] X. Wu, Mon. Not. R. Astron. Soc. 316, 299 (2000); X. 
Wu, T. Chiueh, L. Fang, and Y. Xue, Mon. Not. R. Astron. Soc. 301, 861 (1998); I. Smail et al, Astrophys. J. 479, 70 (1997). 